\title{
CT pulmonary angiography findings in HIV-infected patients referred for suspected pulmonary thrombo-embolic disease
}

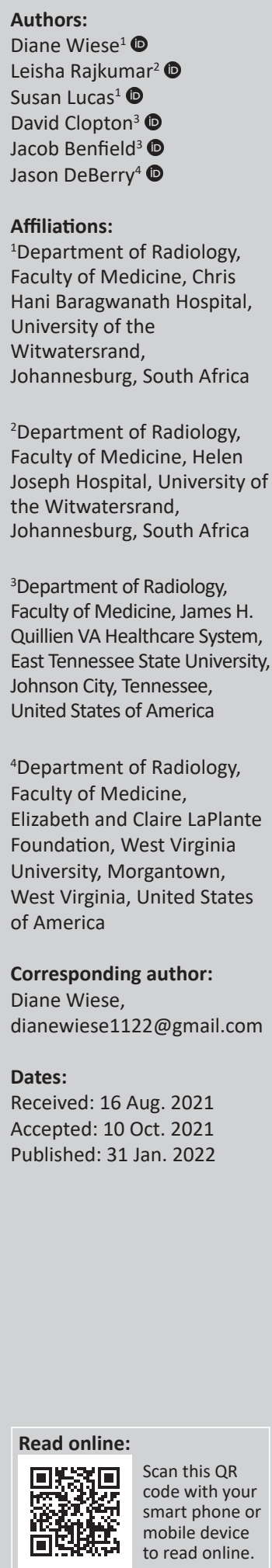

Background: South Africa bares a significant burden of HIV and imaging is commonly performed as part of the workup for respiratory distress.

Objectives: The aim of this study was to document the prevalence of pulmonary thromboembolic disease (PTED) and other findings in HIV-infected patients referred for CT pulmonary angiography (CTPA) for suspected PTED.

Method: Forty CTPA studies of documented HIV-infected individuals investigated for suspected PTED during a 1-year period were retrieved, anonymised and interpreted by three consultant radiologists. Inter-reader reliability was calculated using Free Marginal multi-rater Kappa.

Results: Fourteen of the forty cases (35\%) were positive for PTED. In the pulmonary embolism (PE)-positive group, $57.14 \%$ had peripheral disease and $42.86 \%$ had both peripheral and central disease. Associated findings in the PE-positive cases were pulmonary infarcts (17.5\%), mosaic attenuation (17.5\%) and linear atelectasis (7.5\%). The most common incidental findings were solid pulmonary nodules $(52.5 \%)$, non-wedge-shaped consolidation (45\%), cardiomegaly $(52.5 \%)$ and enlarged intra-thoracic lymph nodes $(52.5 \%)$. Thirty per cent of the study population had findings related directly to the presence of PTED, whilst most cases in the study $(77.5 \%)$ had pulmonary findings unrelated to PTED. In the PE-negative cases, 55\% reported emergent findings that warranted immediate or urgent medical attention.

Conclusion: Computed tomography pulmonary angiography imaging is critical for diagnosing PE. However, further investigation into the judicious application of CTPA in HIV-infected patients with suspected PTED is required, as CTPA findings in most of the cases in this study were unrelated to PE.

Keywords: CTPA; HIV; pulmonary embolism; imaging findings; radiological; prevalence.

\section{Introduction}

Multiple aetiological factors account for respiratory distress in the population affected by HIV, and many pathologies encountered share signs and symptoms of pulmonary embolism (PE). In the clinical setting of this study, CT pulmonary angiography (CTPA) is frequently requested in the workup of respiratory distress in these individuals to exclude PE. HIV has been reported to increase the risk of venous thrombo-embolism (VTE) by 2-10 fold, with a frequency reaching up to $7.6 \%$ per year. ${ }^{1} \mathrm{~A}$ retrospective study in Johannesburg, South Africa showed HIV to be the commonest associated risk factor for VTE. ${ }^{2}$

The general prevalence of PE is estimated to be 600000 cases per year in the United States, ${ }^{3}$ ranging between $0.14 \%$ and $61.5 \%$ in medical patients in different African countries. ${ }^{4}$ Mortality is largely preventable, and thus, diagnosis of PE in hospitalised patients remains paramount to patient care and outcomes. A positive case of PE with parenchymal and pleural complications is shown in Figure 1b.

Paradoxically, studies of CTPA findings for suspected PE have also demonstrated that many alternate findings do not provide a strong rationale for its increased use. ${ }^{5,6} \mathrm{~A}$ study conducted in Brazil in 2016 revealed that a significant proportion of patients who had undergone CTPA for suspected PE had reported alternate findings compatible with an different diagnosis. ${ }^{7}$ This is a relevant consideration for resource-poor settings such as that of South Africa.

How to cite this article: Wiese D, Rajkumar L, Lucas S, Clopton D, Benfield J, DeBerry J. CT pulmonary angiography findings in HIV-infected patients referred for suspected pulmonary thrombo-embolic disease. S Afr J Rad. 2022;26(1), a2273. https://doi.org/10.4102/sajr.v26i1.2273 Copyright: @ 2022. The Authors. Licensee: AOSIS. This work is licensed under the Creative Commons Attribution License. 

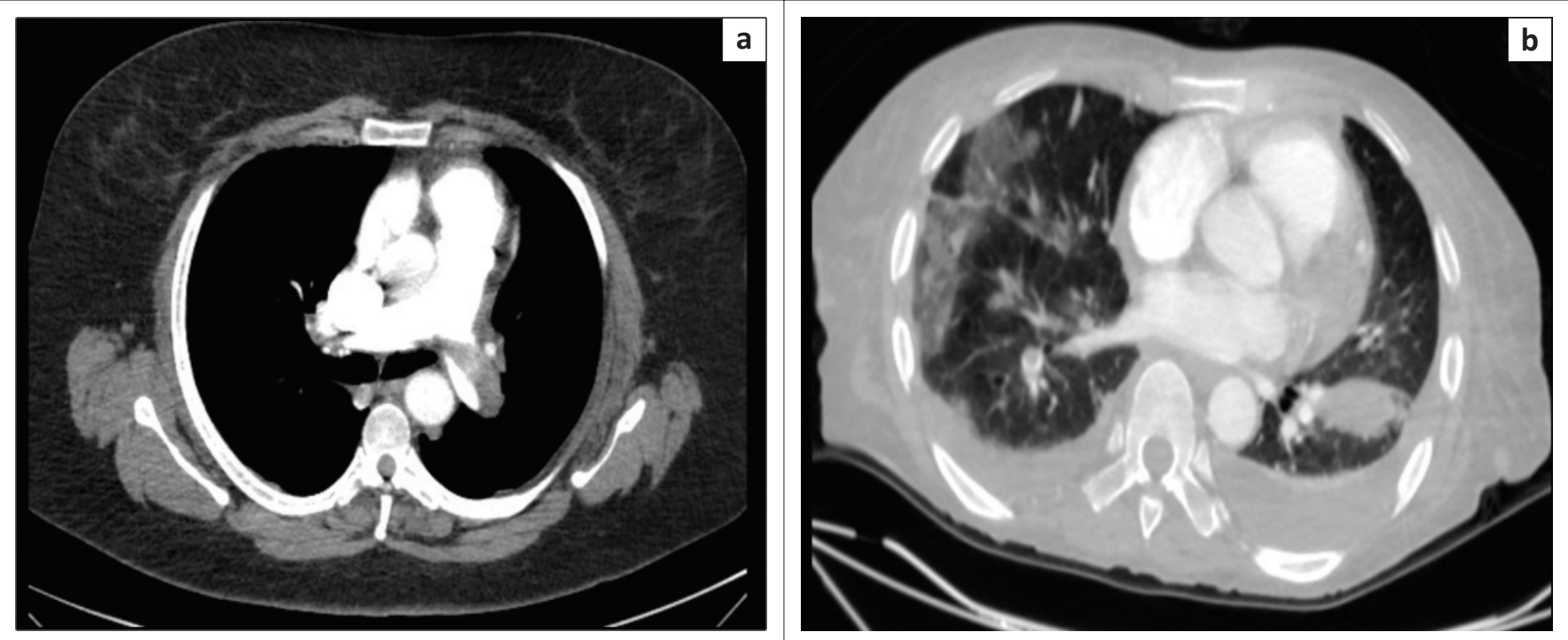

FIGURE 1: Axial CT of two different patients with pulmonary embolism. (a) Demonstrating a large saddle embolus; (b) demonstrating a filling defect in the right descending pulmonary artery complicated by bilateral pleural effusions, peripheral consolidations and a wedge-shaped pulmonary infarct on the left.

The aim of this study was to document the CT findings and the CTPA findings for the prevalence of pulmonary thromboembolic disease (PTED) in HIV-infected patients, given that limited data on this exist in the current literature and CTPAs are frequently ordered for the workup of respiratory distress in these individuals, which contributes further to the currently overloaded healthcare system.

\section{Methods}

A cross-sectional, retrospective, descriptive study was conducted in the Department of Radiology at Helen Joseph Hospital, based in Johannesburg, South Africa. Convenience sampling was utilised whereby all adult HIV-infected patients who had undergone a CTPA for suspected PTED during the study period (January-December 2018) were considered for inclusion in the study. CTPA records with inaccessible digital imaging or request forms with illegible information were excluded.

Patient demographics and presenting symptoms were collected from the CTPA request forms. The HIV status was collected from request forms and/or the National Health Laboratory Service database. Patient identifiers were removed from the CTPA studies using anonymisation software. Patients in the study were scanned on a Phillips 16 slice CT scanner. $100 \mathrm{~mL}$ Omnipaque 350 was injected via 18 $\mathrm{G}$ or $20 \mathrm{G}$ intravenous cannulas in the antecubital fossa using an automated pressure injector at a rate of four millilitres per second, followed immediately by a $50 \mathrm{~mL}$ saline bolus chaser.

The anonymised studies were read by three radiologists, each with experience exceeding four years. Readers assessed 15 criteria via questionnaire-format tick sheets for each CTPA study on Research Electronic Data Capture (REDCap). The study finding descriptors are detailed in Table 1. CT pulmonary angiography findings were classified as PEpositive by a reader based on the presence of pulmonary
TABLE 1: Description of terms.

\begin{tabular}{|c|c|}
\hline Term & Description/defined as \\
\hline Parenchymal & $\begin{array}{l}\text { Findings limited to lung parenchyma excluding pulmonary } \\
\text { vessels and lymph nodes }\end{array}$ \\
\hline Pleural & Findings limited to visceral and parietal pleura \\
\hline Cardiac & Findings limited to heart and pericardium \\
\hline RV:LV & $\begin{array}{l}\text { Presence of right heart strain recorded as present if the } \\
\text { value exceeded } 1^{18}\end{array}$ \\
\hline MPA:AA & PAH recorded as present if the value exceeded $1: 1^{19}$ \\
\hline Extra thoracic & $\begin{array}{l}\text { Findings included soft tissue, visceral, bone and nodal } \\
\text { findings above the thoracic inlet or below the diaphragm }\end{array}$ \\
\hline Other intra-thoracic & $\begin{array}{l}\text { Findings included abnormalities of mediastinal or hilar } \\
\text { lymph nodes, oesophagus, bronchial arteries and anterior } \\
\text { mediastinum }\end{array}$ \\
\hline Emergent findings & $\begin{array}{l}\text { Recorded as present if findings other than PE were present } \\
\text { on the study that the reader deemed severe enough to } \\
\text { cause respiratory distress that would warrant urgent or } \\
\text { immediate medical attention. Examples provided in the tick } \\
\text { sheet included pneumothorax, massive pleural effusion, } \\
\text { extensive consolidation, cardiac failure, significant } \\
\text { pericardial effusion, aortic dissection, haemorrhaging } \\
\text { aneurysm, massive ascites, severe abdominal disease, } \\
\text { tracheal or central bronchial obstruction) }\end{array}$ \\
\hline
\end{tabular}

$\mathrm{RV}: \mathrm{LV}$, right ventricle to left ventricle ratio; MPA:AA, ratio of main pulmonary artery diameter to ascending aorta diameter; $\mathrm{PAH}$, pulmonary arterial hypertension; $\mathrm{PE}$, pulmonary embolism.

artery filling defects and determined overall to be PE positive if two of the three readers agreed it was positive.

Descriptive data in terms of frequencies and percentages were logged for all findings present using SAS 9.2 statistical software. Inter-rater reliability (using Randolph's Free Marginal multi-rater Kappa) was utilised to investigate the level of agreement between the three readers.

\section{Ethical considerations}

This study was approved by the Human Research Ethics Committee (Medical) of the University of the Witwatersrand, reference number M200256.

\section{Results}

A total of 241 CTPAs were identified for the study period, of which 179 were excluded based on a negative or unknown 
HIV status, 2 were also excluded due to illegible request forms and 20 due to irretrievable digital images. The final analytical sample consisted of 40 patients. Fourteen of the 40 patients (35\%) who underwent CTPA had reported PTED. The 95\% confidence interval for this population proportion was between $20.63 \%$ and $51.58 \%$.

The mean and median ages overall were 39 and 38 years, respectively, in men, and 42 and 37 years, respectively, in women. The mean and median ages of PE-positive cases were 40 and 42 years, respectively, in men, and 38 and 37 years, respectively, in women. There was a higher frequency of women compared with men, with a total of 27 women overall $(67.5 \%)$. Of the 14 PE-positive cases, nine $(64.3 \%)$ were women. The major presenting symptoms included shortness of breath $(29.85 \%)$, chest pain $(8.23 \%)$, cough $(11.8 \%)$ and haemoptysis $(8.8 \%)$.

Table 2 provides a detailed summary of the PE-positive CTPA findings. Twenty-seven percent had features of right heart strain, with $22.5 \%$ having a right ventricle to left ventricle $(\mathrm{RV}: \mathrm{LV})$ ratio $>1$.

A complete list of the incidental findings on CTPA is summarised in Table 3 and the pulmonary findings in PEnegative cases are illustrated in Figure 2. The most common parenchymal findings in PE-negative cases were as follows: solid pulmonary nodules $(52.5 \%)$, non-wedge-shaped consolidation (45\%) (Figure 2a, b, c), emphysematous changes (17.5\%) (Figure 2d) and lung cavities (10\%) (Figure $2 \mathrm{e}, 2 \mathrm{f})$. Pleural effusion was also found in $17 \%$ of PEnegative studies (Figure 3). One of the patients in the study had a pneumothorax (Figure 4). The most common incidental cardiac finding was cardiomegaly (52.5\%). Various examples of extra-pulmonary incidental findings are demonstrated in Figure 5, including a case of extensive

TABLE 2: CTPA findings in pulmonary embolism positive cases.

\begin{tabular}{|c|c|c|}
\hline PE positive findings & Frequency & Percentage \\
\hline \multicolumn{3}{|l|}{ Pattern of involvement } \\
\hline Peripheral & 8 & 57.14 \\
\hline Central and peripheral & 6 & 42.86 \\
\hline \multicolumn{3}{|l|}{ Lobe affected } \\
\hline RUL & 5 & 12.50 \\
\hline RML & 5 & 12.50 \\
\hline RLL & 8 & 20.00 \\
\hline LUL & 5 & 12.50 \\
\hline LLL & 5 & 12.50 \\
\hline All (saddle embolus) & 2 & 5.00 \\
\hline \multicolumn{3}{|l|}{ Parenchymal findings } \\
\hline Pulmonary infarct & 7 & 17.50 \\
\hline Mosaic attenuation & 7 & 17.50 \\
\hline Linear atelectasis & 3 & 7.00 \\
\hline Presence of pleural effusion & 8 & 57.14 \\
\hline Presence of right heart strain (RV:LV > 1) & 9 & 22.50 \\
\hline $\begin{array}{l}\text { Evidence of PAH } \\
\text { (MPA:AA > 1:1) }\end{array}$ & 7 & 50.00 \\
\hline
\end{tabular}

pneumobilia (Figure 5b) and a patient found to have multiple rib fractures (Figure 5c). The most common other intra-thoracic finding in PE-negative cases was enlarged mediastinal or hilar lymph nodes without significant mass effect $(52.5 \%)$, whilst $7.5 \%$ had reported an oesophageal abnormality (Figure 5a); the most common extra-thoracic finding was hiatus hernia $(7.5 \%)$.

The readers were $86.67 \%$ agreeable that of the PE-negative patients in the study, 55\% had emergent findings that warranted immediate or urgent medical attention. Thirty percent of the study population also had associated findings related directly to the presence of $\mathrm{PE}$ and $77.5 \%$ had pulmonary findings unrelated to PE (Table 4). In terms of inter-rater reliability, the readers had a 'good-toexcellent' overall agreement, with Kappa exceeding 0.60. Specifically, for 12 out of the 15 criteria assessed, the readers had an excellent overall agreement, with Kappa exceeding 0.75 .

\section{Discussion}

South Africa has one of the highest HIV burdens worldwide. Statistics in South Africa indicates the estimated total

TABLE 3: Incidental findings on CTPA for HIV-infected patients referred for suspected pulmonary thrombo-embolic disease.

\begin{tabular}{|c|c|c|}
\hline Incidental findings & Frequency & Percentage \\
\hline \multicolumn{3}{|l|}{ Parenchymal } \\
\hline Non-wedge-shaped consolidation & 18 & 45.00 \\
\hline Solid pulmonary nodules (>3 $\mathrm{mm}$ ) & 21 & 52.50 \\
\hline $\begin{array}{l}\text { Sub-solid pulmonary nodules } \\
(>6 \mathrm{~mm})\end{array}$ & 3 & 7.50 \\
\hline Ground-glass opacities & 8 & 20.00 \\
\hline Emphysematous changes & 7 & 17.50 \\
\hline Lung cavities & 4 & 10.00 \\
\hline Lung cysts & 2 & 5.00 \\
\hline Tree-in-bud & 1 & 2.50 \\
\hline Pulmonary mass/es & 1 & 2.50 \\
\hline \multicolumn{3}{|l|}{ Pleural } \\
\hline Pleural effusion in the absence of PE & 7 & 17.50 \\
\hline Apical pleural thickening & 2 & 5.00 \\
\hline Pneumothorax & 1 & 2.50 \\
\hline \multicolumn{3}{|l|}{ Cardiac } \\
\hline Cardiomegaly & 21 & 52.50 \\
\hline Pericardial effusion & 1 & 2.50 \\
\hline \multicolumn{3}{|l|}{ Other intra-thoracic } \\
\hline Adenopathy without significant mass effect & 21 & 52.50 \\
\hline Adenopathy with significant mass effect & 2 & 5.00 \\
\hline $\begin{array}{l}\text { Oesophageal abnormality (thickening or } \\
\text { dilatation) }\end{array}$ & 3 & 7.50 \\
\hline \multicolumn{3}{|l|}{ Extra-thoracic } \\
\hline Hiatus hernia & 3 & 7.50 \\
\hline Massive ascites & 2 & 5.00 \\
\hline Benign abdominal visceral lesion/s & 2 & 5.00 \\
\hline Acute severe abdominal pathology $\dagger$ & 1 & 2.50 \\
\hline Vertebral or rib fractures & 2 & 5.00 \\
\hline $\begin{array}{l}\text { Vertebral or rib lesions suspicious for } \\
\text { metastases }\end{array}$ & 1 & 2.50 \\
\hline Breast mass suspicious for neoplasm & 1 & 2.50 \\
\hline $\begin{array}{l}\text { Significant but discrete axillary or } \\
\text { supraclavicular nodes }\end{array}$ & 2 & 5.00 \\
\hline Intra-abdominal nodal masses & 1 & 2.50 \\
\hline
\end{tabular}

$P E$, pulmonary embolus.

$\dagger$, demonstrated extensive pneumobilia. 
population infected with HIV in 2020 to be $13 \%$ with a steady increase of cases (from 3.8 million in 2002 to 7.8 million in 2020). ${ }^{8}$ One-fifth of those affected are women in their reproductive ages (15-49 years). ${ }^{8}$ A lowered immune system renders these individuals susceptible to a myriad of typical and atypical diseases, including bacterial pneumonias, mycobacterium tuberculosis (TB), pneumocystis pneumonia (PCP), malignancies and PE. ${ }^{1}$ Pathology encountered on imaging was therefore expected to be severe or atypical, and indeed, this study demonstrated many such cases. Figure 2 (c and f) illustrates diffuse ground glass opacities and cavitary disease with tree-in-bud nodules respectively imaging findings found commonly in patients affected by atypical fungal infections such as PCP or atypical mycobacterial infections such as TB.

In terms of demographics, most patients with PE in this study were women and the average age in men and women with PE ranged from 38 to 42 years. This finding shows that $\mathrm{PE}$ is seen two decades earlier than in non-HIV patients where the mean age of presentation is 67-62 years. 5,6,7,9 This is in keeping with other literature on HIV-infected patients where the mean ages for PE were younger than the general population, ranging from 40 to 45 years. . $3,30,11,12^{\text {Presenting }}$ symptoms in this study were typical of that described in the Prospective Investigation of Pulmonary Embolism Diagnosis (PIOPED II) trial, including shortness of breath, syncope, chest pain and haemoptysis - all of which are non-specific. ${ }^{13}$

The CTPA prevalence of PE in an HIV-infected study population in this study was found to be $35 \%$. A few previous studies on the HIV prevalence in PE-positive patients have reported much lower percentages in the general population of other countries, ranging from $9.5 \%$ to $24.6 \% .^{5,6,7,9,14}$ This indicates that CTPAs are, indeed, a relevant investigation in the evaluation of respiratory distress in both HIV-infected and HIV-negative patients.

Pulmonary thrombo-embolic disease is commonly described as being 'central' or 'peripheral' in terms of location. A study conducted in China by Zhu et al. in 2012 specifically assessed the anatomical distribution of emboli on CTPAs in patients with suspected PE; they reported the central and peripheral (mixed) pattern in 55.6\% and the peripheral pattern in $40.9 \% .^{15}$ The current study found more patients with a peripheral pattern $(57.14 \%)$ compared to the mixed pattern (42.86\%). Zhu et al also demonstrated that the right lung was more affected than the left and the lower lobes more than the upper lobes. ${ }^{15}$ This was echoed in a South African analysis, where lower lobes were also more commonly affected compared with upper lobes in HIV-infected patients. ${ }^{11}$ We reported the RLL to be most affected $(20 \%)$ with a near even distribution of PE within the other lobes (Table 2), the reasons for this not being clear.

Pleural effusion was present in 57\% of the PE-positive cases in this study. This is higher than the $45 \%$ in the study on HIVinfected patients by Ramlakhan et al. ${ }^{11}$, as well as higher than the $25.8 \%$ in the study by Sharma et al. ${ }^{14}$ which did not include patients' HIV status. The most common parenchymal findings in our PE-positive group were pulmonary infarcts and mosaic attenuation $(17.5 \%$ each), which is comparable with other

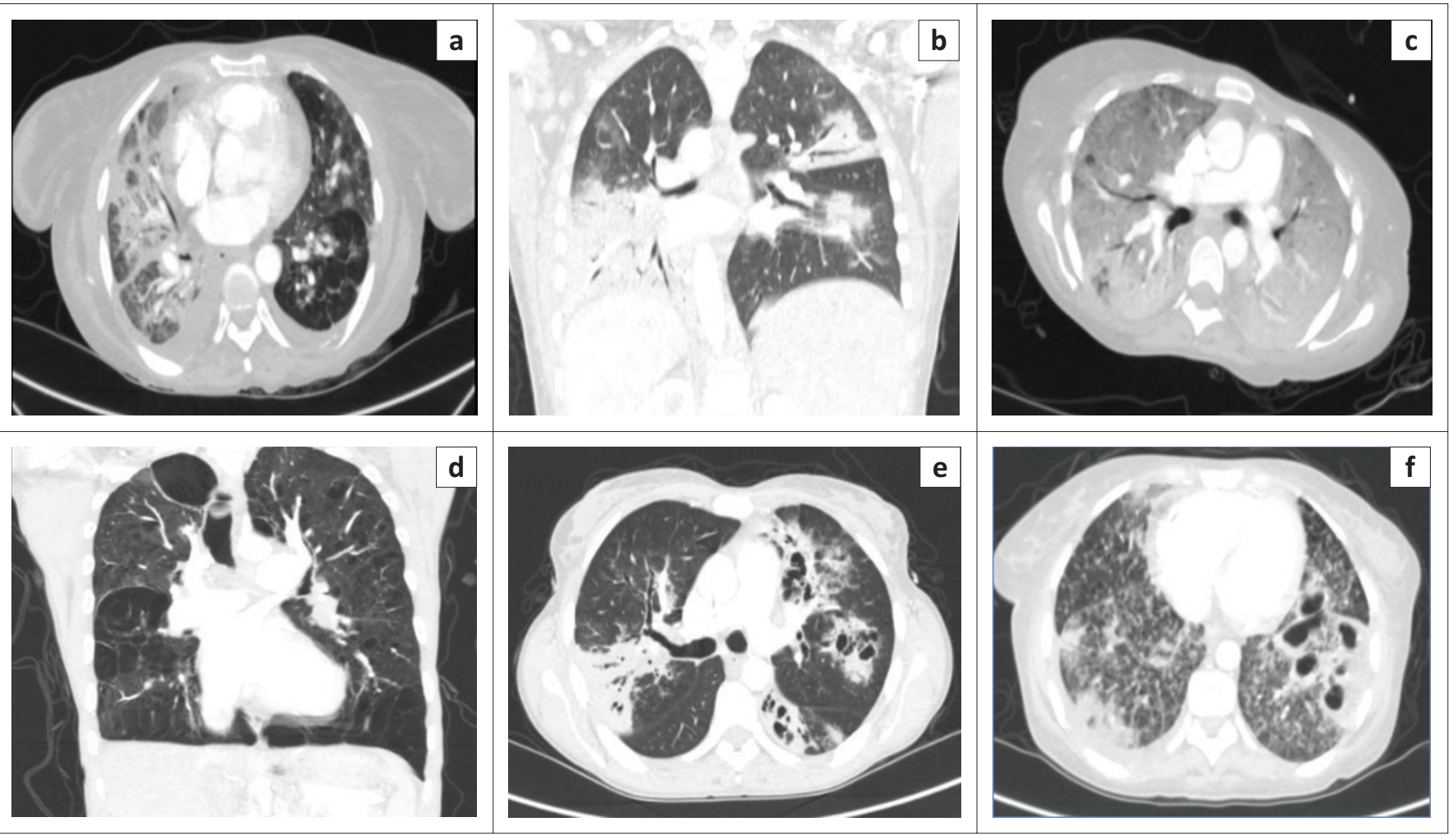

FIGURE 2: Axial ( $a, c, e, f)$ and coronal (b, d) CT slices of incidental parenchymal findings in patients without pulmonary embolism. (a-c) Extensive consolidations in three different patients, (d) diffuse emphysema, (e) cavitary multi-lobar pneumonia, and (f) left lower lobe lung cavitation with bilateral background tree-in-bud nodules in a patient with pulmonary tuberculosis. 
studies carried out on both HIV-infected and -negative populations. ${ }^{7,11,14}$ These findings are traditionally thought to be due to PE. This study demonstrated an increased RV:LV ratio in nine of the 14 PE-positive patients (22.5\%). There are no available data in the literature on the frequency of this finding in an HIV-infected study population with PE to compare with.

While our PE findings did not deviate substantially from reported literature on general populations, the incidental findings in this study were quite varied and many were

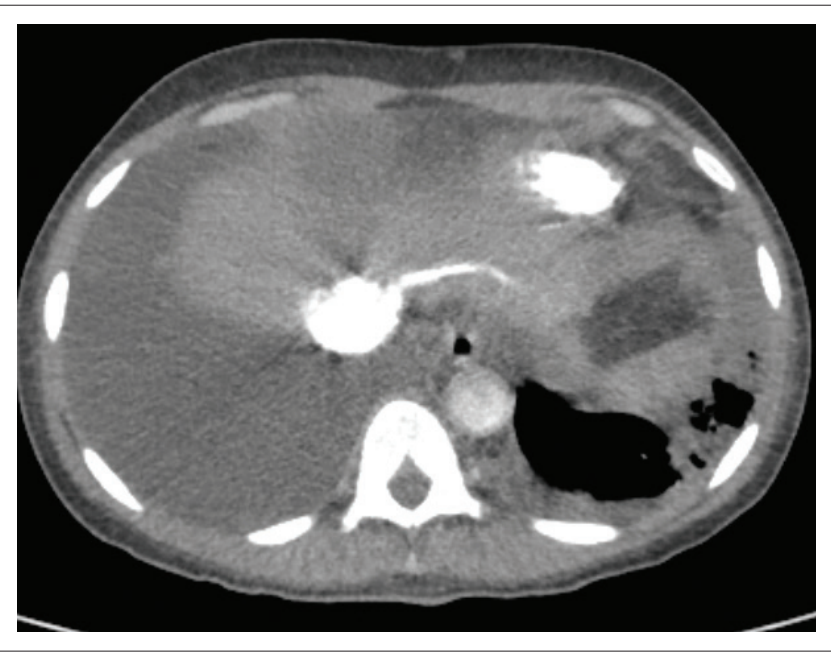

FIGURE 3: Axial CT of a patient with a large right and smaller left pleural effusion.

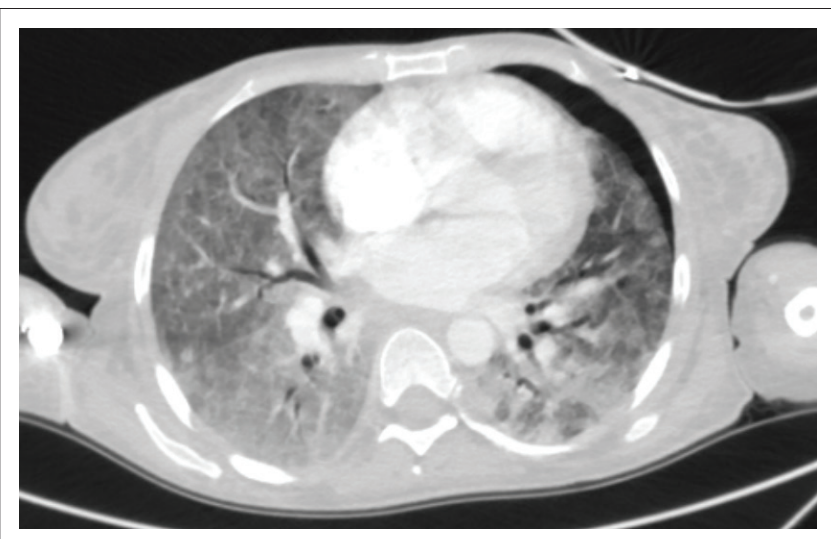

FIGURE 4: Left pneumothorax, extensive ground-glass opacities and posterior consolidations in a patient with respiratory distress. significant. Most of the cases in this study (77.5\%) had reported abnormal lung parenchymal findings unrelated to $\mathrm{PE}$, consistent with other literature. ${ }^{7,9,11,14,16}$ These findings are expected in immunocompromised individuals presenting with respiratory distress.

Importantly, it was demonstrated that of the 26 PE-negative cases, 55\% had emergent findings that warranted urgent medical attention (Table 4). This is significantly higher than other studies, ${ }^{6,7,9}$ and may, in part, be because of the injudicious use of CTPAs whereby patients with respiratory distress are referred for CTPA even when the diagnosis may not likely be that of PE. It is expected that the severity of lung changes encountered in this study is likely to have been more extensive than that found in studies on HIV-negative populations. Previous studies in general populations demonstrated lower percentages of major findings in PE-negative scans. ${ }^{6,7,9}$ However, the current study was limited by the smaller sample size.

The prevalence of non-wedge-shaped consolidation in this study (45\%) was higher than similar studies carried out by Ferreira et al. $^{7}$ and Sharma et al. $^{14}(21.5 \%$ and $25 \%$, respectively). These two studies examined incidental findings on CTPA for suspected PE in general populations (not HIV specific), which may account for the differences seen. The current study also documented a lower prevalence of nonwedge-shaped consolidation (45\%) when compared with the $68 \%$ reported by Ramlakhan et al. which was undertaken in the Western Cape in a region known with a high TB prevalence. ${ }^{11}$ Only one case in this study was rated normal

TABLE 4: Frequency and percentages for the presence of emergent findings and study impression.

\begin{tabular}{lcc}
\hline Emergent findings & Frequency & Percentage \\
\hline Study impression & 22 & 55.0 \\
Findings related to the presence of PE & 12 & 30.0 \\
Pulmonary findings not related to PE & 31 & 77.5 \\
Cardiac findings not related to PE & 4 & 10.0 \\
Other significant intra-thoracic findings & 2 & 5.0 \\
(mediastinal/nodal/oesophageal) not related to PE & & \\
Extra-thoracic significant findings & 1 & 2.5 \\
Normal study & 1 & 2.5 \\
\hline
\end{tabular}

Note: frequency $(\mathrm{n})$, percentage $(\%)$

$\mathrm{PE}$, pulmonary embolus.
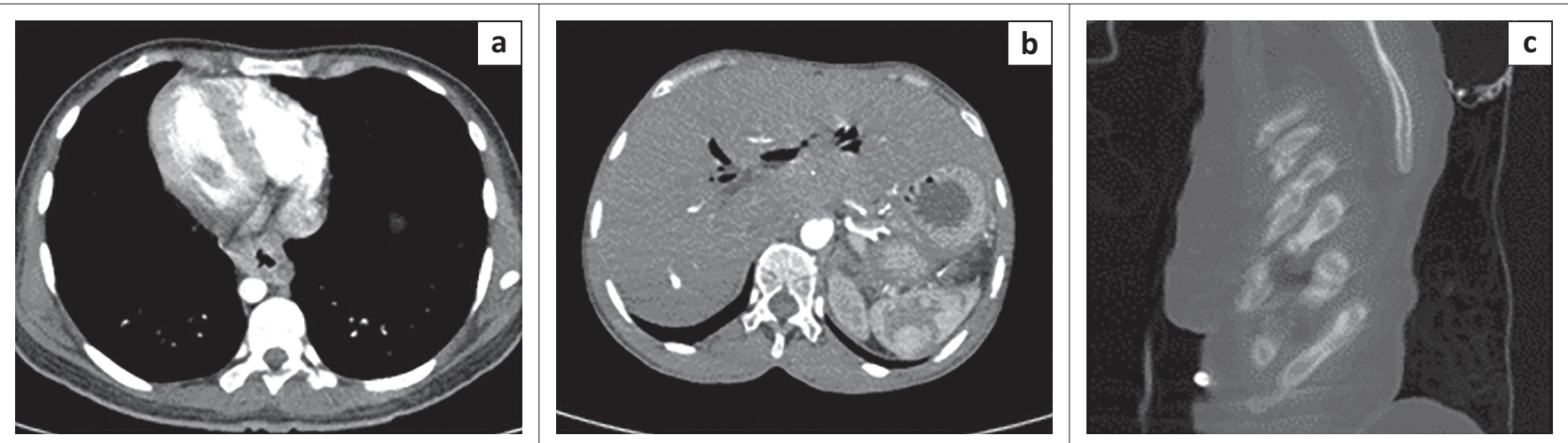

FIGURE 5: Axial (a, b) and sagittal (c) CT images of incidental extra-pulmonary findings in three pulmonary embolism-negative patients. (a) A patient with circumferential lower oesophageal wall thickening, (b) extensive pneumobilia in a post-surgical patient and (c) patient with multiple chronic rib fractures. 
$(2.5 \%)$ with no alternate findings, which is lower than that found in other studies. ${ }^{17}$

While some findings did not require immediate medical attention, they were still significant in that their presence would have likely resulted in further workup or follow-up. Obvious limitations exist in this study. Due to its retrospective nature and small sample size, this study was mainly descriptive.

\section{Conclusion}

Pulmonary embolism is a cause of respiratory distress in HIV-infected patients and CTPA imaging is the gold standard for evaluation. However, further investigations into the prudent use and the efficacy of PE-risk determining scores should be performed regarding the request of CTPAs. HIV-infected patients presenting with symptoms of respiratory distress are not always straightforward PE cases with a multitude of other important pathologies incidentally encountered on CTPA imaging. While there is an obvious benefit to detecting a variety of emergent conditions on CTPA, further investigation into whether such conditions found in this study could be determined on other appropriate investigations, such as chest radiographs, will be of value in curbing the widespread application of CTPAs.

\section{Recommendation}

Consideration for a future study would be to compare the chest radiograph findings in HIV-infected patients referred for suspected PE to their CTPA findings to correlate if findings on initial radiographs and CTPAs lead to markedly different diagnoses and whether this affects the treatment outcome in any significant way.

\section{Acknowledgements Competing interests}

The authors declare that they have no financial or personal relationships that may have inappropriately influenced them in writing this article.

\section{Authors' contributions}

S.L. and L.R. conceived the original idea and supervised the project. D.W. developed the study design, data collection and analysis. D.C., J.B. and J.D. interpreted and analysed the data. D.W. took the lead in writing the manuscript with S.L. and L.R., supervising and contributing to the final version submitted, which was approved by all authors.

\section{Funding information}

This research received no special grant from any funding agency in the public, commercial or not-for-profit sectors.

\section{Data availability}

The data supporting the findings of this study are available from the corresponding author, D.W.

\section{Disclaimer}

The views and opinions expressed in this article are those of the authors and do not necessarily reflect the official policy or position of any affiliated agency of the authors.

\section{References}

1. Bibas M, Biava G, Antinori A. HIV-associated venous thromboembolism. Mediterr J Hematol Infect Dis. 2011;3(1):e2011030. https://doi.org/10.4084/mjhid.2011.030

2. Goldstein LN, Wu M-T. A one year audit of patients with venous thromboembolism presenting to a tertiary hospital in Johannesburg, South Africa. Afr J Emerg Med. 2018;8(1):12-15. https://doi.org/10.1016/j.afjem.2017.08.006

3. Meel S, Peter A, Menezes $C$. The clinical features and management of pulmonary embolism at Chris Hani Baragwanath Academic Hospital. Afr J Thoracic Crit Care Med. 2018;24(3):113-117.

4. Danwang C, Temgoua MN, Agbor VN, Tankeu AT, Noubiap JJ. Epidemiology of venous thromboembolism in Africa: A systematic review. J Thromb Haemost. 2017;15(9):1770-1781. https://doi.org/10.1111/jth.13769

5. Chandra S, Sarkar PK, Chandra D, Ginsberg NE, Cohen RI. Finding an alternative diagnosis does not justify increased use of CT-pulmonary angiography. BMC Pulm Med. 2013;13:9. https://doi.org/10.1186/1471-2466-13-9

6. Perelas A, Dimou A, Saenz A, et al. Incidental findings on computed tomography angiography in patients evaluated for pulmonary embolism. Ann Am Thorac Soc. 2015;12(5):689-695. https://doi.org/10.1513/AnnalsATS.201404-1440C

7. Ferreira EV, Gazzana MB, Sarmento MB, et al. Alternative diagnoses based on CT angiography of the chest in patients with suspected pulmonary thromboembolism. J Bras Pneumol. 2016;42(1):35-41. https://doi.org/10.1590/S1806-37562016000 000105

8. Statistics South Africa. Mid-year population estimates, 2020 [serial online] [cited 2 Jun 2021]. Publication 0302. Available from: http://www.statssa.gov. za/?page_id=1854\&PPN=P0302\&SCH=72634

9. Anjum O, Bleeker H, Ohle R. Computed tomography for suspected pulmonary embolism results in a large number of non-significant incidental findings and follow-up investigations. Emerg Radiol. 2019;26(1):29-35. https://doi.org/ 10.1007/s10140-018-1641-8

10. Ogeng'o JA, Obimbo MM, Olabu BO, Gatonga PM, Ong'era D. Pulmonary thromboembolism in an East African tertiary referral hospital. J Thromb Thrombolysis. 2011;32(3):386-391. https://doi.org/10.1007/s11239-011-0607-4

11. Ramlakhan R, Andronikou S, Rajkumar A. The prevalence and radiological findings of pulmonary embolism in HIV-positive patients referred for computed tomography pulmonary angiography in the Western Cape of South Africa. Cardiovasc J Afr. 2017;28(4):221-228. https://doi.org/10.5830/CVJA-2016-083

12. Bulajic B, Welzel T, Vallabh K. Clinical presentation and diagnostic work up of suspected pulmonary embolism in a district hospital emergency centre serving a high HIV/TB burden population. Afr J Emerg Med. 2019;9(3):134-139. https://doi. org/10.1016/j.afjem.2019.05.003

13. Miniati M, Cenci C, Monti S, Poli D. Clinical presentation of acute pulmonary embolism: Survey of 800 cases. PLoS One. 2012;7(2):e30891. https://doi.org/ 10.1371/journal.pone.0030891

14. Sharma S, Lucas CD. Increasing use of CTPA for the investigation of suspected pulmonary embolism. Postgrad Med. 2017;129(2):193-197. https://doi.org/10.1 080/00325481.2017.1281084

15. Zhu L, Wang J-G, Liu M, et al. Anatomic distribution of embolus at CT pulmonary angiography in patients suspected acute pulmonary embolism. Zhonghua Jie He He Hu Xi Za Zhi. 2012;35(11):833-836.

16. Perelas A, Dimou A, Saenz A, et al. Incidental findings on computed tomography angiography in patients evaluated for pulmonary embolism. Ann Am Thorac Soc. 2015;12(5):689-695. https://doi.org/10.1513/AnnalsATS.201404-144OC

17. Venkatesh SK, Wang SC. Central clot score at computed tomography as a predictor of 30-day mortality after acute pulmonary embolism. Ann Acad Med Singap. 2010;39(6):442-447.

18. Ghaye B, Ghuysen A, Bruyere P-J, D'Orio V, Dondelinger RF. Can CT pulmonary angiography allow assessment of severity and prognosis in patients presenting with pulmonary embolism? What the radiologist needs to know. RadioGraphics. 2006;26(1):23-39. https://doi.org/10.1148/rg.261055062

19. Castañer E, Gallardo X, Ballesteros E, et al. CT diagnosis of chronic pulmonary thromboembolism. RadioGraphics. 2009;29(1):31-50. https://doi.org/10.1148/rg. 291085061 